\title{
Mayak and the Typology of Labial Harmony
}

\author{
Adam G. McCollum \\ University of California, San Diego
}

\section{Introduction}

Labial harmony exhibits a number of restrictions on both triggers and targets, which has prompted a variety of theoretical analyses (e.g. Steriade 1981; Cole \& Trigo1988; Mester 1988; Kaun 1995, 2004; Jurgec 2011; Moskal 2012, van der Hulst \& Moskal 2013, McCollum 2016). Of these, Kaun (1995, 2004) develops the most systematic cross-linguistic treatment of labial harmony, arguing that labial harmony is motivated by perceptual similarity, where more similar (i.e. less salient) contrasts are the best triggers for harmony. In addition, she contends that the best targets for harmony involve the greatest perceptual salience. The Optimality theoretic (OT; Prince \& Smolensky 1993/2004) implementation of her analysis encodes five asymmetric preferences cross-linguistically, shown below.
Kaun's $(1995,2004)$ typological generalizations with accompanying constraints
1. The trigger must be [-hi].
ALIGN-L/R([RD]/[-HI])
2. The trigger must be [-bk].
ALIGN-L/R([RD]/[-BK])
3. The trigger must be short.
ALIGN-L/R([RD]// $\left.\mathrm{V}_{\mu}\right)$
4. The target must be [+hi].
$*[-\mathrm{HI},+\mathrm{RD}]$
5. The trigger and target must agree in height.
GestURAL Uniformity[RD]

One empirical prediction that falls out from these generalizations is that cross-height harmony should not preferentially target non-high vowels. As first noted in Steriade (1981), this accounts for a notable height asymmetry in Turkic labial harmony. This is evident in Kyrgyz, as described in Hebert \& Poppe (1963; cf. Comrie 1981), shown below in (2). Observe in (2a-d) that if the root (i.e. leftmost) vowel is [-bk], then progressive labial harmony is obeyed regardless of trigger or target height. In contrast, among [+bk] root vowels, as in (2e-h), if the trigger is [+high] and the target is [-high], then labial harmony does not obtain, as demonstrated in $(2 \mathrm{~h})$

Labial harmony in Kyrgyz (Hebert \& Poppe 1963)
$\begin{array}{lllll}\text { a. køz-dy } & \text { 'eye-ACC' } & \text { e. } & \text { koj-du } & \\ \text { b. køz-dø } & \text { 'eye-LOC' } & \text { f. } & \text { koj-do } & \text { 'sheep-ACC' } \\ \text { c. kyz-dy } & \text { 'autumn-ACC' } & \text { g. tuz-du } & \text { 'sheep-LOC' } \\ \text { d. kyz-dø } & \text { 'autumn-LOC' } & \text { h. tuz-da *tuz-do } & \text { 'salt-ACC' } \\ \text { isalt-LOC' }\end{array}$

Examples like those in (2) lend strong support to Kaun's analysis, as Kyrgyz exemplifies four of Kaun's five constraints, ALIGN-R([RD]/[-HI]), ALIGN-R([RD]/[-BK]), *[-HI, +RD] and GeSTURAL UNIFORMiTY[RD]. Kyrgyz also demonstrates a key claim in Kaun's work- cross-height harmony on a non-high target is dispreferred. Implicationally, if cross-height harmony targets non-high vowels, then harmony must be unrestricted. This falls out from the preference for high targets, enforced by *[-HI, $+\mathrm{RD}]$, and the preference for same-height harmony, enforced by GESTURAL UNIFORMITY[RD].

*I am grateful for helpful discussion with Sharon Rose, Anna Mai, Kati Hout, UCSD's PhonCo, as well as interaction with the audience at AMP 2016. I would also like to thank Doris Payne for sharing the Maa data with me. All errors are my own. 
However, labial harmony in Mayak (Andersen 1999a), counter-exemplifies the claim that there is a universal preference for non-high targets. In Mayak, labial harmony proceeds regressively, triggering alternations in non-high vowels. In (3a,b), the non-high root vowel, $/ \Lambda /$, optionally undergoes rounding to [o] before a round vowel. If this type of harmony, which Kaun's analysis regards as the most marked type, occurs in the language, then harmony should be unrestricted. This is not the case, though. In $(3 \mathrm{c}, \mathrm{d})$, the high root vowels, /i/ and /I/, do not undergo rounding.

$$
\begin{aligned}
& \text { Labial harmony in Mayak } \\
& \text { a. tık-uð-i } \sim \text { tok-uð-i 'wash.AP-PST-SUF' } \\
& \text { b. ?^m-uð-i ?om-uð-i 'eat.AP-PST-SUF' } \\
& \text { c. ?ið-u *?uð-u 'shape.with.axe-PST' } \\
& \text { d. mry-øk *moy-ok 'spider-PL' }
\end{aligned}
$$

In this paper I argue three things: first, Mayak is exceptional under Kaun's set of constraints, second, despite apparent exceptionality, Mayak does not invalidate Kaun's claim that labial harmony is perceptionmotivated, and third, by redefining similarity in terms of perceptual similarity offers a new way of analyzing the Mayak pattern and the larger typology of labial harmony. By mediating a definition of similarity through distinctive features Kaun's analysis can only indirectly encode perceptual similarity. Instead, by defining perceptual similarity in dispersionary terms (Liljencrants \& Lindblom 1972), I demonstrate that both the Mayak case and the larger typology of harmony fall out from similarity, but similarity recast in auditory, not featural, terms.

\section{Mayak}

Mayak is a Western Nilotic language spoken primarily in South Sudan, also known as Burun. The data provided here comes from Andersen (1999a, personal communication). Mayak has an inventory of eight phonemic vowels, /i i $\varepsilon$ a $\supset \mho \mathrm{u}$ /, with two additional surface vowels, [e o]. In addition to regressive labial harmony, Mayak also exhibits bidirectional ATR harmony (see Finley 2007; Trommer 2016 for analyses). Regressive ATR harmony, which is the most robust harmony pattern in the language, triggers alternations

\begin{tabular}{|c|c|c|c|c|}
\hline \\
\hline \multicolumn{5}{|c|}{$\begin{array}{l}\text { Regressive ATR harmony } \\
\text { Root vowel }\end{array}$} \\
\hline a. & Root vowel & Pamb- $\Lambda$ & Pamb-ir & 'eat' \\
\hline b. & $\begin{array}{l}\mathrm{a} \\
\varepsilon\end{array}$ & geb- $\Lambda \mathrm{r}$ & geb-ir & 'beat' \\
\hline $\begin{array}{l}0 . \\
\text { c. }\end{array}$ & 0 & nond- $\Lambda \mathrm{r}$ & nond-ir & 'fold' \\
\hline \multirow{2}{*}{$\begin{array}{l}\text { d. } \\
\text { e. }\end{array}$} & I & PId $-\Lambda \mathrm{r}$ & Pid-ir & 'shape with axe' \\
\hline & v & god- $\Lambda \mathrm{r}$ & gud-ir & 'untie' \\
\hline \multirow{2}{*}{$\begin{array}{l}\text { f. } \\
\text { g. }\end{array}$} & $\Lambda$ & $\mathrm{p} \Lambda \mathrm{d}-\Lambda \mathrm{r}$ & $\mathrm{p} \wedge \mathrm{d}-\mathrm{ir}$ & 'untie' \\
\hline & $\mathrm{i}$ & Pib- $\Lambda \mathrm{r}$ & Pib-ir & 'shoot' \\
\hline h. & $\mathrm{u}$ & tuJ- $-\Lambda \mathrm{r}$ & tuJ-ir & 'send' \\
\hline
\end{tabular}
in non-low vowels when preceded by $[+\mathrm{hi},+\mathrm{ATR}]$ vowels. These are demonstrated below, in (4). Note that non-low [-ATR] roots undergo assimilation in (4b-e), but that low, (4a), and [+ATR] roots, (4f-h), retain their underlying ATR features.

From the data in (4) we can conclude that $\varepsilon$-e,,$-\mathrm{o}$, I-i, and $\mho-\mathrm{u}$ are surface ATR pairs. As for the vowels, /a/ and $/ \Lambda /$, these two vowels are paired only in progressive ATR harmony. The singulative suffix is underlyingly /a/, but surfaces as $[\Lambda]$ after $[+$ ATR] vowels, shown in (5). This is the only direct source of evidence that $/ \mathrm{a} /$ and $/ \Lambda /$ form a harmonic pair. 
(5)

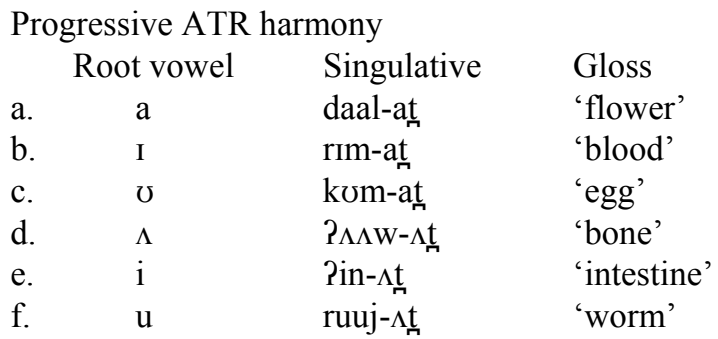

As noted above, labial harmony operates on non-high vowels when followed by round vowels. As in (3), the data in $(6 a, b)$ show that the non-high vowel, $/ \Lambda /$ surfaces as [o] before $[u]$. In contrast, high vowels do not undergo rounding before [+hi, +rd] vowels, demonstrated in $(6 \mathrm{c}, \mathrm{d})$. Additionally, $(6 \mathrm{e}-\mathrm{g})$ show that the vowels, / $\varepsilon$ a $\Lambda$ / do not undergo harmony before $/ \mathrm{s}$. Positive evidence for harmony is only available from forms like $(6 a, b)$, where $[u]$ triggers rounding of $/ \Lambda /{ }^{1}$

\begin{tabular}{|c|c|c|}
\hline 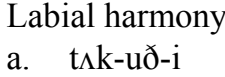 & $\sim$ tok-uð-i & 'wash.AP-PST-SUF' \\
\hline b. ?Аm-uð-i & Рom-uð-i & 'eat.AP-PST-SUF' \\
\hline Pið-u & *?uð-u & 'shape.with.axe-PST' \\
\hline miz-ひk & *moy-øk & 'spider-PL' \\
\hline Wil-ol & *wol-ol & 'guest-SG' \\
\hline wely-on & *woly-on & 'rib-SG' \\
\hline maað-эnюn & *məวð-эnวn & 'drink-1P.EXC' \\
\hline PAt-onэn & *?ot-onon & 'pull.CNTRF-1P.EXC \\
\hline
\end{tabular}

There are no instances of non-initial [o] due to a positional restriction in the language, so we cannot conclusively rule out [o] as a potential trigger for harmony. Also, the disharmony evident in (6e-h) may not derive from the inertness of $/ \mathrm{s} /$ as a trigger of harmony. Rather disharmony may stem from the inability of /I $\varepsilon$ a/ to undergo harmony in (6e-f). As for (6h), underlying / $/$ does not surface as [o] because progressive ATR harmony does not target mid vowels. Moreover, both progressive and regressive ATR harmony involve [+ATR] spreading, so the [-ATR] vowel, [0], is not a possible trigger for ATR harmony in $(6 \mathrm{~g}, \mathrm{~h})$. It is argued throughout the literature that labial harmony is parasitic on some other form of harmony or vowel agreement (Mester 1988; Cole \& Trigo 1988; Jurgec 2011; van der Hulst \& Moskal 2013; McCollum 2016). If this is the case, then labial harmony depends upon ATR harmony. As such, (6h) does not undergo labial harmony because the trigger and target do not agree in ATR. Throughout I will assume that all $[+\mathrm{rd}]$ vowels are intrinsically potential triggers for harmony, but that the parasitic nature of labial harmony precludes other manifestations of harmony in Mayak. This assumption entails that the restrictions on harmony fall out from restrictions on what vowels may undergo assimilation.

Under this assumption, Kaun's constraint set cannot account for the Mayak data. Crucially, we would need a constraint to privilege non-high targets, which runs afoul of the fourth generalization in (1), namely, the target must be high. If one replaces $*[-\mathrm{HI},+\mathrm{RD}]$, the constraint that penalizes non-high round vowels generally, with some ad hoc constraint to penalize high round vowels, this results in a loss in empirical coverage, and potentially undermines the larger analysis set forth by Kaun. Instead, I show in the following section that the phonetic underpinnings of Kaun's analysis still offer insight into the Mayak labial harmony, but necessitate a more phonetically-mediated constraint set.

\section{Perceptual similarity and Dispersion theory}

3.1 Perceptual similarity in Kaun (1995, 2004) Two phonetic studies of similarity figure prominently into Kaun's analysis, Linker (1982) and Terbeek (1977). Linker (1982) examines the articulatory characteristics of round vowels in five languages. She suggests that lip protrusion and lip

\footnotetext{
${ }^{1}$ There is a second progressive ATR harmony pattern that targets only [+hi] vowels.
} 
compression are the most salient correlates of lip rounding. Overall, she finds that back vowels and high vowels exhibit more lip rounding than front and non-high vowels, respectively. Importantly, though, the lip positions and the degree of articulatory difference for round-unround contrast, like e- $\varnothing$, differ across languages. Thus, the particular lip gestures are not uniform. Terbeek's (1977) study demonstrates that high vowels and back vowels are perceived as more rounded than non-high vowels and front vowels, parallel to Linker's production study. However, Terbeek's analysis also shows significant cross-linguistic variation over the degree of perceptual distinctiveness between round-unround pairs. As such, Kaun's interpretation of these studies constitutes a reasonable generalization across-languages, but language-internally, the specific gestures that manifest lip rounding the perceptual cues of lip rounding differ to some degree.

Language-specifically, Mayak counter-exemplifies Kaun's extrapolation from these studies, not because lip rounding is fundamentally different in Mayak. We cannot say for sure, but it seems unlikely that this is the case. Rather, it seems more appropriate to argue that perception is contingent, not on the universal properties of a given vowel, but rather on the relationship between that vowel and relevant members of the inventory in which it occurs. While Linker and Terbeek's studies were contextualized to specific languages, Kaun's abstraction over human language implies that all $/ \mathrm{u} / \mathrm{vowels}$ should be more rounded and by extension, more perceptually distinct from all $/ \mathrm{y} /$ or $/ \mathrm{o} /$ vowels, for example. This prediction simply does not follow from these studies. Rather, it is more likely that perceptual similarity hinges upon system-internal factors, which are not considered in Kaun's analysis.

3.2 Dispersion theory Dispersion theory (DT; Liljencrants \& Lindblom 1972; Lindblom 1986; Schwartz et al. 1997; de Boer 2001; Padgett \& Tabain 2005 a.o.) casts the fitness of a given inventory in terms of the perceptual distances separating each member of the inventory from every other member. DT relies on acoustic data to predict the distribution of vowels across a large number of inventories, with relative success, especially on languages with seven or fewer vowels.

DT becomes most relevant for our discussion when we localize the notion of perceptual distinctiveness to individual contrasts. DT provides a language-specific metric through which to evaluate the nature of contrast without necessary reference to abstract features. It is important to note that more phoneticallybased representations do not obviate the need for features. Rather, I suggest herein that a richer array of information is available to speakers, and from this array, certain phonetic properties govern the likelihood that a vowel will participate in labial harmony.

Like previous work in DT, I operationalize contrast in terms of weighted Euclidean distances between vowels. The domain within which these measurements are made is the acoustic F1-F2 vowel space. Although F3 is also commonly associated with lip rounding, McCollum (2016) finds that F1-F2 provide a better fit to data in several Eurasian languages, and I therefore use distance in a two-dimensional space to define similarity. This involves several steps. First, raw acoustic data is transformed into a psychoacoustic scale to more closely approximate vowel perception. In this study I converted mean F1-F2 from Hertz to ERB (Equivalent Rectangular Bandwidth), as in Padgett \& Tabain (2005). Second, the range of F2 contrasts is compressed, resulting in a vowel space where the range of F2 contrasts to F1 contrasts is 0.50.75:1. This warping reflects the more general perceptual salience of F1 over F2 contrasts (see Schwartz et al. 1997; Padgett \& Tabain 2005). As in McCollum (2016), I chose to compress the F2:F1 vowel space to $0.625: 1$, the middle value in the proposed range. To do this, the target ratio, .625:1, is divided by the range of attested F2:F1 contrasts to give the warping factor, $\lambda$. This warping factor, $\lambda$, is then used to compute the weighted Euclidean distance, $\delta$, between two vowels, $x$ and $y$, using the formula in (7).

Formula for weighted Euclidean distances between harmonic pairs, $x$ and $y$

$$
\delta_{x y}=\sqrt{\left(F 1_{x}-F 1_{y}\right)^{2}+\lambda^{2}\left(F 2_{x}-F 2_{y}\right)^{2}}
$$

Given a set of harmonic pairings, a-b, c-d, e-f...x-y, the above equation returns a numerical distance for each pair. This distance can be directly compared to other pairings in the language to determine which pairings are more perceptually similar (i.e. smaller distances) and which are not. The upshot of this quantification of contrast is that it makes clear, falsifiable predictions about the relative likelihood a given round vowel will initiate and undergo harmony. 
3.3 Dispersion-based predictions Using this DT metric of contrast localized to individual contrasts allows us to recast Kaun's typological predictions. If we assume the Euclidean distance between two pairs, $x$ and $y$, is $\delta_{x y}$, then Kaun's general idea that perceptual similarity motivates harmony can be reformulated thusly: vowel pairings separated by smaller Euclidean distances will be more likely to trigger harmony. This prediction is formalized via an alignment constraint, shown in (8). The constraints used herein separate the set of participants from non-participants using a categorical boundary, $m$, shown below. ${ }^{2}$

$$
\operatorname{ALIGN-L([RD]/\delta _{xy}<m)-}
$$

align the feature [rd] to the left edge of the word if the distance between the $[\mathrm{rd}]$ trigger, $x$, and its harmonic pair $y, \delta_{x y}$, is less than $\mathrm{m}$.

Kaun curtails spreading with conditional alignment constraints, framed similarly to (8) above, as well as two markedness constraints, *[-HI, +RD] and GESTURAL UNIFORMITY[RD]. These limit non-high round vowels and cross-height spreading, respectively. Kaun argues that high round vowels are preferred targets for harmony because they are more perceptually salient, and thus better cues to the roundness of the initial trigger. In contrast, the present analysis assumes that a vowel's likelihood of undergoing harmony is conditioned upon its relationship to its surface harmonic pair, where more similar contrasts are more likely to undergo alternations, following Steriade (2001; Zuraw 2007, 2013; Hayes \& White 2015). This is encoded in the formalism via a surface markedness constraint banning alternations that exceed some categorical boundary, $n$.

$$
* \operatorname{MAP}\left([\mathrm{RD}] / \delta_{x y}>n\right)-
$$

assign a violation to every output [rd] alternation between vowels $x$ and $y$ that involves a Euclidean distance, $\delta_{x y}$, greater than $\mathrm{n}$.

The constraints in (8) and (9) generate the prediction that within a given inventory the vowel(s) most likely to trigger harmony will also be the most likely to undergo harmony. This parallels the formulation of GESTURAL UNIFORMITY[RD] in Kaun's analysis, but falls out from these two constraints without necessary reference to trigger-target relations. ${ }^{3}$

\section{Determining Euclidean distances}

In order to implement this framework we need acoustic data from Mayak. However, no Mayak speakers were found outside of (South) Sudan, and due to the current geopolitical situation, travelling to this area of Africa to collect data was not feasible. Thus, in the absence of the necessary data, this section develops two possible ways of quantifying contrast, either by using Flemming's (2002) auditory representations of the vowel space, or by using data from a related language, Maa (Guion et al. 2004).

4.1 Flemming's auditory representations Flemming's (2002) idealized grid, shown in Figure 1 below, offers one possible solution to the lack of Mayak data. Note that this representational schema offers little room for language-specific nuances in vowel quality. Also, this representation crucially hinges on, typically impressionistic, transcriptions. This dependence on transcriptions is aggravated by transcription practices that make little reference to surface vowel quality, but rather use a more abstract notion of featural contrast. Given these limitations, using Flemming's auditory representations is not ideal, but I demonstrate below that, despite these limitations, Flemming's schema works for this particular language. In Figure 1, the surface vowel inventory of Mayak is in black while other vowel qualities used by Flemming are in gray.

\footnotetext{
${ }^{2}$ A gradient version of this constraint is used in McCollum (2016).

${ }^{3}$ GeStURAL UnIFORMity[RD], in some form, is still probably necessary for an analysis of Kyrgyz. This is briefly discussed in $\S 6.3$.
} 
Figure 1: Auditory representations in Flemming (2002:30)

\begin{tabular}{|c|c|c|c|c|c|}
\hline 5 & 4 & 3 & 2 & 1 & $\mathrm{~F} 2 / \mathrm{F} 1$ \\
\hline $\mathrm{i}$ & $\mathrm{y}$ & $\dot{\mathrm{t}}$ & u & $\mathrm{u}$ & 1 \\
\hline I & $\mathrm{Y}$ & & & U & 2 \\
\hline \multirow[t]{3}{*}{ (e) } & $\varnothing$ & $\partial$ & $\gamma$ & (o) & 3 \\
\hline & $\varepsilon$ & $œ$ & $\Lambda$ & 0 & 4 \\
\hline & & $\mathrm{a}$ & $a$ & & 5 \\
\hline
\end{tabular}

To make this representational schema useful, it is first necessary to compress the F2 dimension by 0.625 , since the current grid make the range of F2:F1 contrasts $1: 1$ rather than the proposed $0.625: 1$. After doing this, we need only measure distances between each of the possible round-unround pairs in the language. The possible pairings and their respective distances are shown below in Table 1.

The distances in Table 1 accord with the predictions made above, namely that the most similar roundunround pairing is most likely to participate in harmony. In Table 1, observe that the surface pairing, $\Lambda$-o, is separated by the smallest distance. Using this, we could construct an analysis where targets are restricted such that only the least perceptually salient pair(s) may alternate for harmony, and all other pairings in the language are too dissimilar to undergo harmonic alternations.

Table 1: Possible [rd] harmonic pairings and their respective distances with Flemming's grid

\begin{tabular}{|c|c|}
\hline Pairing & Perceptual Distance \\
\hline$\Lambda-\mathrm{O}$ & 1.18 \\
\hline $\mathrm{a}-\mathrm{\jmath}$ & 1.6 \\
\hline$\varepsilon-\mathrm{\jmath}$ & 1.88 \\
\hline $\mathrm{e}-\mathrm{O}$ & 2.5 \\
\hline $\mathrm{i}-\mathrm{u}$ & 2.5 \\
\hline $\mathrm{I}-\mho$ & 2.5 \\
\hline
\end{tabular}

This, however, hinges on $/ \Lambda /$ being realized with a lower F1 and F2 than $/ \mathrm{a} /$. . There are two pieces of evidence to support that this is actually the case in Mayak- the general realization of the [+ATR] low vowels in Nilotic more generally and two relevant descriptions of the language (Andersen 1999a;.Hall \& Creider 1998). In Nilotic, there are a number of languages with a [+ATR] low vowel, including Mayak and the other related languages (Andersen 1999b, 2004, 2007). Hall \& Creider show that in several southern Nilotic languages, including Nandi and Keyyo, that the [+ATR] low vowel is actually raised and backed toward [o]. Strikingly, in Nandi there is almost complete surface neutralization of these underlying vowels. Thus, one common trend in Nilotic is for the [+ATR] low vowel, transcribed in Andersen (1999a) as / $\mathrm{N}$ to be raised and sometimes backed in the acoustic space.

More important, though, are the two descriptions of the low [+ATR] vowel by Hall \& Creider (1998) and Andersen (1999a). Hall \& Creider report that Hall \& Hall's field notes have this vowel transcribed as $[\Lambda]$ in Mayak, as $[\gamma] \sim[\Lambda]$ in closely related Mabaan, and $[\gamma] \sim[u]$ in another related language, Jumjum. Andersen corroborates these impressions, noting that "phonetically, $[\Lambda]$ is less open and more back than [a], but as will become clear...it is structurally the [+ATR] counterpart of [a], so it must be classified as a low central vowel" (1999a:2). This same point is reiterated later in the paper (1999a:13). Therefore, using Flemming's grid, which represents $/ \Lambda /$ as both higher and farther back than $/ a /$ is justified for Mayak. It remains to be seen how effective Flemming's grid is for analyzing labial harmony more generally.

4.2 Data from Maa While Flemming's schema works for the analysis of Mayak, it does so by abstracting away from the very language-specific properties I want to highlight. Therefore, I demonstrate below that the vowel qualities in Maa, a related Nilotic language, also point to surface similarity as a defining force in the labial harmony pattern in Mayak. 
Guion et al. (2004) show that the Maa inventory has only nine surface vowels, with the [+ATR] low vowel acoustically neutralized to its [-ATR] harmonic counterpart. Data from Guion et al. (2004) was graciously shared by Doris Payne. This data was then used to plot the mean and standard deviations for the Maa vowel inventory, shown below in Figure 2. ${ }^{4}$ Mean F1-F2 in Hz and ERB is presented in Table 2. The Mayak-specific value of $\lambda, .571$, was calculated by dividing .625 by 1.095 , the ratio of F2 to F1 ranges in Maa, (5.87 ERB for F2, and 5.36 ERB for F1).

Figure 2: Maa vowel plot with means and 1-SD ellipses $(\mathrm{N}=2,066)$

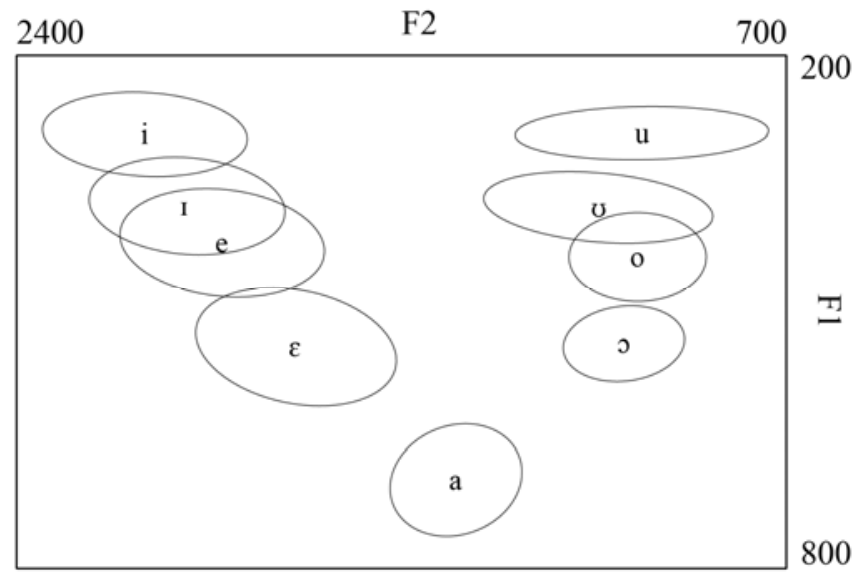

Table 2: Mean F1-F2 for Maa vowels with range of F1 and F2 over mean vowel qualities

\begin{tabular}{|c|c|c|c|c|}
\hline Vowel & Mean F1 (Hz) & Mean F1 (ERB) & Mean F2 (Hz) & Mean F2 (ERB) \\
\hline $\mathrm{a}$ & 697 & 12.99 & 1443 & 18.48 \\
\hline $\mathrm{o}$ & 538 & 11.24 & 1059 & 16.06 \\
\hline $\mathrm{o}$ & 436 & 9.91 & 1029 & 15.84 \\
\hline $\mathrm{U}$ & 377 & 9.05 & 1115 & 16.45 \\
\hline $\mathrm{u}$ & 291 & 7.63 & 1019 & 15.76 \\
\hline$\varepsilon$ & 541 & 11.28 & 1783 & 20.20 \\
\hline $\mathrm{e}$ & 419 & 9.67 & 1948 & 20.94 \\
\hline $\mathrm{I}$ & 376 & 9.03 & 2024 & 21.25 \\
\hline $\mathrm{i}$ & 292 & 7.64 & 2117 & 21.63 \\
\hline
\end{tabular}

Using the data in Table 2, along with our language-specific $\lambda$, 0.571, we can determine the Euclidean distances between each harmonic pair. Before doing this, though, we must propose some F1-F2 values for $\mathrm{I} / \mathrm{t}$ to reflect what Andersen reports about this vowel in the language. If we use the relatively conservative values, 575 and 1250 for F1 and F2, which in turn equate to 11.68 and $17.34 \mathrm{ERB}$, we obtain the distances in Table 3 using the equation from (7). These distances assume that other than $/ \mathrm{N} /$, the vowel qualities in Mayak are identical to those in Maa.

Table 3 shows that the proposed placement of $/ \Lambda /$, situated in between $/ a /$ and $/ \mathrm{o} /$, gives us distances in which the attested harmonic pairing is more similar than other pairings in the language. One thing to note at this point is that only ATR-harmonic pairings were considered, as also in Table 1 . The $\Lambda$ - $\supset$ pairing would have been the most similar without this restriction, but in the presence of a pervasive ATR harmony dictating that $/ \mathrm{N} /$ may not be realized as [0]. The role of ATR harmony is discussed in the analysis presented in the next section.

\footnotetext{
${ }^{4}$ Maa data in this paper from Doris Payne were collected with partial support from NSF grant SBR-9809387 awarded to the University of Oregon.
} 
Table 3: Proposed Euclidean distances for Mayak from Maa data

\begin{tabular}{|c|c|}
\hline Vowel Pairing & Distance (ERB) \\
\hline$\Lambda-\mathrm{o}$ & 1.97 \\
\hline $\mathrm{a}-\mathrm{O}$ & 2.23 \\
\hline$\varepsilon-\mathrm{O}$ & 2.36 \\
\hline $\mathrm{I}-\circlearrowright$ & 2.74 \\
\hline e-o & 2.92 \\
\hline $\mathrm{i}-\mathrm{u}$ & 3.35 \\
\hline
\end{tabular}

\section{Formal analysis}

Using the weighted Euclidean distances above from Maa, we can formalize harmony in Mayak using a relatively small set of constraints. Above we saw the two crucial dispersionary constraints used in the analysis, ALIGN-L $([\mathrm{RD}] / \delta<\mathrm{m})$, and $* \operatorname{MAP}([\mathrm{RD}] / \delta>\mathrm{n})$. In addition to these two constraints we need a constraint to implement ATR harmony in Mayak. There are multiple ways to implement the parasitic relationship between labial and ATR harmony in Mayak. Jurgec (2011) analyzes parasitic assimilation in terms of a conditional AGREE constraint that enforces agreement for the feature, F, only when agreement for some orthogonal feature, $\mathrm{G}$, is also obeyed. While this possibility more directly encodes the parasitism argue to undergird the presence of labial harmony, this solution increases the complexity of the harmonydriving constraints in a significant way. I have already introduced a conditional harmony-driving constraint, ALIGN-L $([\mathrm{RD}] / \delta<\mathrm{m})$, but if we add a second layer of conditionality on a harmony-driving constraint, there is a significant increase in constraint-internal complexity. If we construct a constraint, AGREE([RD], [ATR]), that says adjacent vowels agree in rounding if they agree in ATR, much like the constraints in Jurgec (2011), we lose the generalization that labial harmony depends on surface similarity. A symmetrical AGREE constraint (Lombardi 1999; Bakovic 2000), fails to capture the asymmetric directionality of harmony in Mayak (Finley 2009), and since the Mayak pattern's directionality cannot derive from some positional prominence, as affixes trigger assimilation of roots for both ATR and labial harmony, there is no straightforward way to model this with a symmetric AGREE constraint.

One other possibility is increasing the complexity of the extant alignment constraint, but as AGREE cannot encode directionality, ALIGN cannot make syntagmatic reference to possible targets. More concretely, a general ALIGN constraint cannot take into account the nature of possible targets, so predicating labial harmony upon successful ATR harmony is not manageable with a single ALIGN constraint, either. I thus employ a separate ALIGN-L constraint enforcing ATR harmony and stipulate that it must outrank ALIGN-L[RD]. The high ranking of this constraint effectively bans labial harmony on ATRdisharmonic sequences, as in (6h) above. Two further details are necessary to set up the analysis. First, we need to define the variables $m$ from the alignment constraint in (8) and $n$, the variable from the *MAP constraint in (9). As for $\mathrm{m}$, I assume that there are no surface similarity-based restriction on triggers, a general ALIGN-L[RD] is sufficient for the analysis. As for $\mathrm{n}$, the threshold beyond which [rd] assimilations are banned. The numerical value is arbitrary, and for the analysis I adopt the value 2.1 because it distinguishes the alternating pair from the non-alternating pairs.

$$
\begin{array}{ll}
* \operatorname{MAP}\left([\mathrm{RD}] / \delta_{x y}>2.1\right)-\quad & \text { assign a violation to every output }[\mathrm{rd}] \text { alternation between vowels } x \text { and } \\
& y \text { that involves a Euclidean distance, } \delta_{x y}, \text { greater than } 2.1 .
\end{array}
$$

If the above *MAP constraint outranks ALIGN-L[RD], we can restrict the set of potential alternants to $[\Lambda]$ [o], as in (11) and (12) below. In (11), ALIGN-L[+ATR] is satisfied because both trigger and target are both underlyingly [+ATR]. *MAP is violated by both Candidates (b) and (c) because in each an [i]-[u] alternation takes place, either as unrounding the trigger in Candidate (b), or rounding the target in Candidate (c). Both of these competitors are eliminated by violations of *MAP, leaving the attested output, Candidate (a), as the winner. 


\begin{tabular}{|c|c|c|c|c|c|c|c|}
\hline & & $/$ Pið-u/ & ALIGN-L[+ATR] & $*$ MAP $(\delta>2.1)$ & ALIGN-L[RD] & $\begin{array}{c}\text { ID-IO } \\
{[\text { ATR] }}\end{array}$ & $\begin{array}{c}\text { ID-IO } \\
{[\text { RD] }}\end{array}$ \\
\hline$*$ & a. & Pið-u & & & $*$ & & \\
\hline & b. & Pið-i & & $* ! \mathrm{i} \sim \mathrm{u}$ & & & $*$ \\
\hline & c. & Puð-u & & $* ! \mathrm{u} \sim \mathrm{i}$ & & & $*$ \\
\hline
\end{tabular}

In (12), we see harmony obtain in tok-uð-i from (6a). Candidate (d) is eliminated by violating ALIGNL[+ATR], while Candidate (c) is ruled out due to a perceptually large alternation necessary to remove the trigger for labial harmony. Candidate (b) does not undergo harmony, and as a result, is ruled out by ALIGN$\mathrm{L}[\mathrm{RD}]$, leaving the attested form, Candidate (a), as the optimal output.

\begin{tabular}{|c|c|c|c|c|c|c|c|}
\hline & & /tık-uð-i// & ALIGN-L[+ATR $]$ & $* \operatorname{MAP}(\delta>2.1)$ & ALIGN-L[RD] & $\begin{array}{c}\text { ID-IO } \\
\text { [ATR] }\end{array}$ & $\begin{array}{c}\text { ID-IO } \\
\text { [RD] }\end{array}$ \\
\hline$\sigma$ & a. & tokuði & & & & & * \\
\hline & b. & tskuði & & & $* !$ & & \\
\hline & c. & tıkiði & & $* ! \mathrm{i} \sim \mathrm{u}$ & & & $*$ \\
\hline & d. & tokuði & $* !$ & & & $*$ & \\
\hline
\end{tabular}

The above analysis encodes perceptual similarity via a *MAP constraint that penalizes surface alternations above the language-specific threshold, 2.1 ERB. I claim herein that there is no one cross-linguistic threshold for similarity, but that languages with labial harmony implement different thresholds that all function in the same way- they promote harmony from perceptually similar contrasts, and penalize surface alternations that involve larger perceptual shifts. As noted above, the dispersionary analysis developed herein and in McCollum (2016) predicts that sequences of identical vowels should be strongly preferred cross-linguistically because the more similar a vowel is to its pair the more likely it will both initiate harmony and surface via harmony. This prediction is further explored in the following section.

\section{The larger typology of labial harmony}

6.1 Gestural Uniformity and syntagmatic identity The dispersionary analysis predicts that sequences of identical vowels should be more typical of labial harmony across the world's languages than other patterns. In contrast, Kaun's analysis does not directly predict this. Trigger-target uniformity is encoded via GESTURAL UNIFORMITY[RD], but as one of a battery of constraints used to motivate her larger analysis, this preference is suppressed under many constraint rankings. To further demonstrate the veracity of the dispersionary claim- that trigger-target uniformity is the most crucial aspect of any typological study of labial harmony, I present statistical evidence from a survey of 61 languages with labial harmony.

Kaun's survey includes 33 languages, of which over 20 are Turkic, the rest being largely Mongolic and Tungusic. This genetic/areal skew is significant, because we now know that many languages outside Eurasia also demonstrate this harmony pattern. So, in the present survey, 61 languages were included. Of those 61, only 29 are Turkic (48\%). Moreover, a number of Bantu languages (Boyd 2015), as well as Kwa languages (e.g. Essegbey 2009; Bobuafor 2013) are included in the study that were absent from Kaun's earlier study. In sum, the survey that forms the basis of the statistical generalizations to follow is almost twice the size of Kaun's survey and is far more genetically diverse.

To assess the relative importance of each of Kaun's constraints, every possible vowel sequence involving a round vowel in a triggering position was considered for these 61 languages. Each sequence was coded for violations to Kaun's constraints along with the attested outcome, either disharmony or harmony. For instance, a sequence u...u in Kyrgyz is attested, but a sequence u...o is not, violating *[-HI, +RD], as well as GESTURAL UNIFORMITY[RD]. The full model included random intercepts for language and language family, as well, to further avoid any skewing due to genetic affiliation. Note that ALIGN-L/R([RD]/V $\mu$ ) was excluded from the model because it is only relevant for a few languages of Eurasia (see Walker 2001; Kaun 2004), leaving only four constraints to examine. 
The full set of vowel sequences, coded for language, language family, actual harmony/disharmony, as well as violations to Kaun's constraints were analyzed using a logistic regression. Then each fixed effect (i.e. Kaun's constraints) were removed one-by-one to test the relative importance of each constraint to fitting the model. The importance of a particular constraint is manifest by the change in AIC (Akaike Information Criterion), $\triangle \mathrm{AIC}$, when compared to the full model. As Burnham \& Anderson (2004:271) note, $\triangle \mathrm{AIC}$ of less than 2 show generally insignificant changes in model fit, whereas $\triangle \mathrm{AIC}$ exceeding 10 show drastic loss in explanatory power without the predictor under consideration. For our purposes, the more significant a given predictor (constraint) is, the greater $\triangle \mathrm{AIC}$ will be when that predictor is removed from the model. Specifically, the dispersionary analysis predicts that $\triangle \mathrm{AIC}$ will be far greater when GESTURAL UNIFORMITY[RD] is removed relative than when other constraints are removed from the full model. The results from these model comparisons are presented in Table 5 below. When *[-HI, $+\mathrm{RD}]$ was removed from the model, both $\triangle \mathrm{AIC}$ and significance testing show a change in model fit trending towards significance. In contrast, when the other three constraints were removed, significant changes in model fit occurred, reflected by $\triangle \mathrm{AIC}>2$ and p-values $<0.05$. Most importantly, the change in model fit when GESTURAL UNIFORMITY[RD] was removed was far larger than any other changes in model fit. The combined $\triangle \mathrm{AIC}$ if the other three models were all removed was 19.27, which is less than half of the effect obtained from removing GeSTURAL UNIFORMity[RD]. In essence, the model hinges upon GeSTURAL UNIFORMITY[RD], while the other constraints are more peripheral. I interpret this as strong confirmation that labial harmony preferentially, and perhaps fundamentally creates syntagmatic vowel identity, which is not explicitly predicted by Kaun's analysis.

Table 5: Nested model comparisons

\begin{tabular}{|l|c|c|c|}
\hline \multicolumn{4}{|c|}{$\mathrm{AIC}_{\text {Full model }}=456.13$} \\
\hline Constraint & AIC without constraint & $\Delta \mathrm{AIC}$ & $\chi^{2}$ test of significance \\
\hline$*[-\mathrm{HI},+\mathrm{RD}]$ & 457.85 & 1.72 & $\chi^{2}(1)=3.73, \mathrm{p}=0.54$ \\
\hline ALIGN-L/R([RD]/[-HI] $])$ & 462.80 & 6.67 & $\chi^{2}(1)=8.67, \mathrm{p}=0.003$ \\
\hline ALIGN-L/R([RD]/[-BK] $])$ & 476.25 & 11.12 & $\chi^{2}(1)=13.12, \mathrm{p}=0.0003$ \\
\hline GESTURAL UNIFORMITY[RD] & 500.64 & 44.51 & $\chi^{2}(1)=46.52, \mathrm{p}=9.09 \times 10^{-12}$ \\
\hline
\end{tabular}

6.2 High vowels One generalization that emerges in McCollum (2016), which is also evident in the Maa data used for the above analysis is that high vowels tend to be more dispersed from one another than non-high vowels. This relates to the claims made by Kaun (1995), namely that high round vowels are more perceptually salient than non-high round vowels. If this is the case, without any other parameters in the dispersionary model, we would predict that vowel height doesn't play a role in labial harmony. This is easily falsifiable, though. Consider Turkish, where high vowels are targeted for harmony while non-high vowels are opaque (Lewis 1967; Underhill 1976). When vowel acoustics are examined, there does not appear to be a clear correlation between perceptual distance and the participation of high vowels in Turkish. For instance, a cursory glance at the Turkish results in Washington (2016), as well as those in Lanfranca (2012) and Türk et al (2004), reveals that the [ \pm rd] high vowels are not more proximate to one another than non-high $[ \pm \mathrm{rd}]$ vowels. How then can this analysis account for the significant skew, not only in Turkish, but also present throughout Turkic, to favor [+hi] targets?

I propose that we need another parameter for vowel duration. Ainsworth (1971) and Hillenbrand et al. (2000) argue that vowel duration significantly affects vowel recognition, particularly in certain regions of the vowel space. If duration is relevant, then the Turkish, and more generally Turkic, problem has a straightforward solution. Turkic languages exhibit a drastic durational difference between high and nonhigh vowels, where high vowels are often subject to complete elision while non-high vowels are more resistant to this reduction process (see Sjoberg 1963; Poppe 1964, 1968; Comrie 1997; Levi 2005; McCollum 2015, 2016; Washington 2016 for further evidence). McCollum (2016) demonstrates that in Kazakh non-high vowels are over twice as long as [+hi] vowels. Therefore, we need only expand the model to include duration as a cue to trigger harmony as well as a perceptual threshold by which to delimit undergoers, as well. This would predict that shorter vowels, like more perceptually similar vowel pairs, would be more likely to trigger and undergo harmony. Assuming that high vowels are also intrinsically 
short in Turkic, there is evidence suggesting that at least one language may instantiate a pattern where short vowels are preferential triggers and targets for harmony, Kachin Khakass (Korn 1969).

6.3 Kyrgyz Thus far the model introduced in McCollum (2016) and herein is likely incapable of handling the Kyrgyz pattern from (1). Without reference to trigger-target relations, it does not seem possible to capture the fact that /a/ maps to [o] after /o/, but not after $/ \mathrm{u} /$. This seems to demand triggertarget relations be directly incorporated into the model. As of yet, they are only indirectly referenced. However, if these are incorporated, phonetic studies of Kyrgyz appear to indicate that the perceptual distance between $/ \mathrm{u} /$ and $/ \mathrm{a} /$ exceeds other palatal-harmonic trigger-target distances (Washington 2006, 2016). This may provide an avenue for further development of the model, although it remains to be seen how much redundancy would inevitably arise in the larger model from such an addition.

\section{Conclusion}

This paper advances three arguments. First, labial harmony in Mayak is exceptional under Kaun's constraint set, as non-high vowels undergo cross-height harmony although high vowels do not. Second, if Kaun's fundamental argument is retained, then a redefinition of contrast in phonetic terms allows for the successful analysis of Mayak. Third, I have demonstrated that the overall predictions of the phoneticallybased dispersionary model presented herein accord with the larger typology of harmony. Specifically, labial harmony tends to result in sequences of identical round vowels (Kaun's GESTURAL UNIFORMITY[RD] constraint), a robust generalization that accounts for far more variance in harmony patterns crosslinguistically than any of Kaun's other predictions.

\section{References}

Ainsworth, William (1972). Duration as a cue in the recognition of synthetic vowels. JASA 51: 648-651.

Andersen, Torben (1999a). Vowel harmony and vowel alternation in Mayak (Western Nilotic). Studies in African Linguistics 28: 1-30.

Andersen, Torben (1999b) Vowel quality alternation in Mabaan and its Western Nilotic history. Journal of African Languages and Linguistics 20: 97-120.

Andersen, Torben (2004). Jumjum phonology. Studies in African Linguistics 33: 133-162.

Andersen, Torben (2007). Kurmuk phonology. Studies in African Linguistics 36:29-90.

Bakovic, Eric (2000). Harmony, dominance and control. PhD dissertation, Rutgers University.

Bobuafor, Mercy (2013). A grammar of Tafi. PhD dissertation, University of Leiden.

de Boer, Bart (2001). The origins of vowel systems. Oxford University Press.

Boyd, Virginia Lee (2015). The phonological systems of the Mbam languages of Cameroon with a focus on vowels and vowel harmony. $\mathrm{PhD}$ dissertation, University of Utrecht.

Burnham, Kenneth P., and David R. Anderson (2004). Multimodel inference understanding AIC and BIC in model selection. Sociological methods \& research 33: 261-304.

Cole, Jennifer, and Loren Trigo (1988). Parasitic harmony. In Features, segmental structure and harmony processes (Part II), van der Hulst \& Smith (eds.) 19-38.

Comrie, Bernard (1981). The languages of the Soviet Union. Cambridge.

Comrie, Bernard (1997). Tatar (Volga Tatar, Kazan Tatar) phonology. Phonologies of Asia and Africa, vol. 2. Alan S. Kaye (ed.). Winona Lake, IN: Eisenbrauns, 899-911.

Essegbey, James (2009). Noun classes in Tutrugbu (Nyagbo). Journal of West African Languages 36, 37-56.

Finley, Sara (2007). Height-Based Restrictions on Vowel Harmony in Mayak. unpublished ms.

Flemming, Edward (2002). Auditory representations in phonology. Routledge.

Guion, Susan G., Mark W. Post, and Doris L. Payne (2004). Phonetic correlates of tongue root vowel contrasts in Maa. Journal of Phonetics 32: 517-542.

Hall, R. M. R., and Chet Creider (1998). The Fates of [+ ATR]/a in Nilotic. In Language History and Linguistic Description in Africa, Ian Maddieson and Thomas J. Hinnebusch (eds.): 45-54.

Hayes, Bruce, and James White (2015). Saltation and the P-map. Phonology 32: 267-302.

Hebert, Raymond J., and Nicholas Poppe (1963). Kirghiz Manual. Uralic and Altaic Series vol. 33. Indiana University.

Hillenbrand, James M., Michael J. Clark, and Robert A. Houde (2000). Some effects of duration on vowel recognition. JASA 108: 3013-3022. 
van der Hulst, Harry, and Beata Moskal (2013). Pattern of defective labial harmony in Turkic languages. In Dilbilim Araştırmaları Dergisi I (Linguistic Investigations. Theme Issue: Updates to Turkish Phonology, 17-52.

Jurgec, Peter (2011). Feature spreading 2.0: A unified theory of assimilation. PhD dissertation, University of Tromsø.

Kaun, Abigail R. (1995). The typology of rounding harmony: an optimality theoretic approach. $\mathrm{PhD}$ dissertation, UCLA.

Kaun, Abigail R. (2004). The typology of rounding harmony. In Phonetically based phonology, Bruce Hayes, Robert Kirchner, and Donca Steriade (eds.): 87-116. Cambridge University Press.

Korn, David (1969). Types of labial vowel harmony in the Turkic languages. Anthropological Linguistics 11: 98-106.

Lanfranca, Mark (2012). An Acoustic Study of Underspecified Vowels in Turkish. MA thesis, University of Kansas.

Levi, Susannah (2005). Acoustic correlates of lexical accent in Turkish. JIPA 35: 73-97.

Lewis, Geoffrey (1967). Turkish grammar. Oxford University Press.

Liljencrants, Johan, and Björn Lindblom (1972). Numerical simulation of vowel quality systems: The role of perceptual contrast. $\operatorname{Lg}$ 48: 839-862.

Lindblom, Björn (1986). Phonetic universals in vowel systems. Experimental phonology, 13-44.

Linker, Wendy. (1982). Articulatory and acoustic correlates of labial activity in vowels: A cross-linguistic study. UCLA Working Papers in Phonetics 56.

Lombardi, Linda. (1999). Positional faithfulness and voicing assimilation in Optimality Theory. NLLT 17: $267-302$.

McCollum, Adam G. (2015). Labial harmonic shift in Kazakh: Mapping the pathways and motivations for change. BLS 41: 329-352.

McCollum, Adam G. (2016). Vowel dispersion and Kazakh labial harmony. San Diego Linguistic Papers 6:29-65.

Mester, Armin (1988). Dependent tier ordering and the OCP. In Features, Segmental Structure, and Harmony Processes, Harry van der Hulst \& Norval Smith (eds): 127-144. Dordrecht: Foris,

Padgett, Jaye, and Marija Tabain (2005). Adaptive dispersion theory and phonological vowel reduction in Russian. Phonetica 62: 14-54.

Poppe, Nicholas (1964). Bashkir Manual: Descriptive grammar and texts with a Bashkir-English glossary. Uralic and Altaic Series. Indiana University.

Poppe, Nicholas (1968). Tatar manual: Descriptive grammar and texts with a Tatar-English glossary, $2^{\text {nd }}$ ed. Uralic and Altaic Series. Indiana University.

Prince, Alan and Paul Smolensky (1993/2004). Optimality Theory: Constraint Interaction in Generative Grammar. Blackwell.

Schwartz, Jean-Luc; Louis-Jean Boe, Nathalie Valee, and Christian Abry (1997). The dispersion-focalization theory of vowel systems. JPh 25: 255-286.

Sjoberg, Andrée (1963). Uzbek structural grammar. Uralic and Altaic Series. Indiana University.

Steriade, Donca (1981). Parameters of metrical harmony rules. unpublished ms.

Terbeek, Dale (1977). A cross-language multidimensional scaling study of vowel perception. PhD dissertation, UCLA.

Trommer, Jochen (2016). Vowel raising in Mayak as compound opacity. Replicative Processes in Grammar, Barnickel, Katja; Matias Guzman Naranjo, Johannes Hein, Sampson Korsah, Andrew Murphy, Ludger Paschen, Zorica Puskar; Joanna Zaleska (eds): 229-258.

Türk, O., Şayli, Ö., Özsoy, A. S., \& Arslan, L. M. (2004). Türkçe'de Ünlülerin Formant Frekans İncelemesi. Paper presented at the 18th Ulusal Dilbilim Kurultay1.

Underhill, Robert (1976). Turkish grammar. MIT Press.

Walker, Rachel (2001). Round licensing, harmony, and bisyllabic triggers in Altaic. Natural Language \& Linguistic Theory 19: 827-878.

Washington, Jonathan North (2006). Root vowels and affix vowels: Height effects in Kyrgyz vowel harmony. unpublished ms.

Washington, Jonathan North (2016). An investigation of vowel anteriority in three Turkic languages using ultrasound tongue imaging. $\mathrm{PhD}$ dissertation, Indiana University.

Zuraw, Kie (2007). The role of phonetic knowledge in phonological patterning: corpus and survey evidence from Tagalog infixation. $\operatorname{Lg}$ 83: 277-316.

Zuraw, Kie (2013). *MAP constraints. unpublished ms. 